\title{
Social Presence Online: Networking Learners at a Distance
}

\author{
Elizabeth Stacey
}

Faculty of Education, Deakin University, 221 Burwood Highway, Burwood, Victoria 3125, Australia estacey@deakin.edu.au

Keywords: collaborative learning, distance learning, pedagogy, higher education

\begin{abstract}
This paper draws on two studies which researched the use of online small group environments where collaborative learning is a central structure for learning. The establishment of social presence is facilitated through the socioaffective aspect of small group interaction which contributed to the effectiveness of learning online. Social presence, the ability of online learners to project themselves into a textual environment which has few visual or contextual cues, will be explored as an important element in facilitating effective online learning. The teacher's role in helping students project their online social presence and in establishing an environment for learning within the larger group computer conference will also be discussed.
\end{abstract}

\section{INTRODUCTION}

"Sometimes we just talked about things not connected to the course at all like the weather, books we were reading and wine we were drinking while studying. These are some of the bonuses of working with computer conferencing. You can develop a good professional and personal rapport with fellow students and facilitators."

"The success of online conferencing seems to lie in the hands of the teacher who 'facilitates' discussions along the way-keeping the momentum going when needed." Students learning online. 
This paper explores the findings of two research studies which focus on the use of small group online environments for collaborative learning. The first study found that the socio-affective support provided by the collaborative group environment was of major importance to the success of the students and provided the basis for the second study which researched the development of this social support system by analysing the establishment of social presence in the course computer conferences. The importance of the teacher's role in helping students project their online social presence and in establishing an environment for learning in a computer conference is also explained.

\section{BACKGROUND OF THE STUDY}

As the online environment becomes more widely used for teaching courses and networking learners, the process of participants establishing social presence in an online environment is beginning to be explored. Social presence, defined by Garrison (1997a) as "the degree to which participants are able to project themselves affectively within the medium", is a growing area of focus for research as teachers and researchers find that for cognitive presence to be sustained, social presence must be established first. In the field of communications technology, which studies social presence as the degree to which communicating participants are able to project their presence through the technology, social presence can be projected best when the verbal and non verbal cues and the context can be also be communicated (Rice 1993). By these criteria, computer mediated communication (CMC) would be judged low on social presence (Lindlif and Shatzer 1998) and the role of the participants in compensating for lack of cues becomes more important.

Walther (1996), writing from the field of communication research, reflected that early empirical studies into $\mathrm{CMC}$ use had concluded that participants would be less sociable and more task oriented and he challenged the notion that $\mathrm{CMC}$ was a depersonalised medium. The results of the studies had been explained theoretically as an effect of the lack of non verbal cues, a perspective called 'cues-filtered-out' (Culnan and Markus 1987). However since this early research, field research has shown that with unconstrained interaction time, participants with high levels of social interaction through email, listserves, internet chat etc. (e.g. Parks and Floyd 1996) provide outcomes which counter the empirical data, though without explaining its results. Walther drew on a social information processing perspective that suggested that as communicators in $\mathrm{CMC}$ are as driven as any participants to develop social relationships, the longer time factor of an 
ongoing computer conference can provide the opportunity for the accrual of interpersonal impressions and the gathering of social information through text messages. In this way relationships can still develop despite fewer cues and the earlier time -limited empirical research results were explained.

Walther tested this theory (Walther 1993, Walther and Burgeon 1992) and found that though interpersonal impressions were formed more slowly with CMC, relationships developed in the same way as in face to face situations, even becoming more socially oriented in the online context.

Though Gunawardena and Zittle (1997) claim that social presence in a CMC context focus on the group members' reactions to one another because the teacher acts as 'more of a facilitator than the main source of information', this paper describes a proactive role the teacher plays in developing a socially responsive discourse that models a combination of social encouragement with content discussion. Garrison has called this aspect of the teacher's successful conference moderation 'contextualising' where the teacher provides the communication model as well as organising and focusing the online discussion (1997b). The criteria analysing social presence involves extending an analysis framework developed for the first study to include social presence factors developed in a study by Rourke, Anderson, Garrison and Archer (1999), which measured social presence. Their work in defining the 'community of inquiry' model that holds the teaching presence as an important factor in structuring learning and facilitating active learning is extended here.

\section{CONTEXT OF THE RESEARCH}

The first study into online collaborative learning of students within a Masters of Business Administration Program (Stacey 1999) found that interactive online group discussion was central to the learners' effective construction of new conceptual understandings. Through the social context of group interaction, the collaborative groups developed a consensus of knowledge through communicating different perspectives, receiving feedback from other students and teachers and discussing ideas, until a final negotiation of understanding was reached. The social nature of cognition as theorised by Vygotsky (1978) and developed through neo-Vygotskian research (Forman and McPhail 1993) was seen as influential to a person's construction of knowledge. The provision of dialogue within a community of learners was of major importance as knowledge construction is perceived as a dialectic process in which individuals test their constructed views on others and negotiate their ideas. Drawing on Vygotsky's theory that conceptual understandings are developed through verbal interaction, this research found 
that a socially constructed learning environment is essential for effective learning. The social conversation provided the learner with a context and stimulus for thought construction and learning which was the means by which the group contributes more to each learners' understanding than they are able to do individually.

The groups were based in a diverse range of workplaces and geographical locations in three states of Australia remote from the delivering university location. The gender balance of the groups represented the overall course population with more males (21) than females (10). The socio-affective support provided by the collaborative group environment was found to be important to the success of the students who established social support systems through their online groups. This support system was particularly vital to them as distance learners who may otherwise be isolated from other students. The posting of supportive comments and sharing of personal anecdotes and information provided a network of social interaction that underlay the mutual respect and trust needed for successful collaborative learning.

Each small group's message content analysis revealed more than $50 \%$ of messages sent online were social in nature, intended to maintain the cohesion of the group and support and encourage the groups' individual members. The social messages gave the students the friendship and sense of belonging that helped to motivate them to apply themselves to their study when they were finding it hard to manage, particularly because of the conditions of studying at a distance. Their accountability and responsibility to the other members of the group for their participation in online collaborative tasks were strong motivators for their persistence and such a sense of a strong group network depended on their establishment of social presence online.

\section{SOCIAL PRESENCE RESEARCH}

The second study was designed to explore these findings and was developed within the content area of an Open and Distance Education specialism of a Masters degree program. This paper focuses on one course called Teaching and Learning with Computer Mediated Communication which was developed in response to students' growing interest in the theory and practice of teaching and learning in a virtual medium. Online conferencing has been used in this program since 1993 with all units having conferences established through the communication software FirstClass, for electronic discussion of ongoing tasks and activities with the aim of engaging students in learning interactively. The small group collaborative 
learning environment was researched to explore how the collaborative learning environment affects the students' learning and within this context, how social presence factors were established online particularly focusing on the role of teacher in modeling techniques of social interaction.

\section{RESEARCH METHODS}

Data were gathered electronically using qualitative methods through archiving and analysing the online interaction of the students and by gathering students' perceptions and reflections. Quantitative data were collected through analysis of the frequency and type of messages on the conferences as well as through content analysis of the computer conferences. The participant group had 20 members.

1. Through the FirstClass unit conferences, a voluntary focus group was set up within a course conference area where questions were posted at the beginning and at the end of the semester. These asked about the students' experience studying online.

2. Another interactive FirstClass conference was established three months later to discuss the early analysis of results and further encourage student reflections on the effect conferencing had on their learning.

3. The main data gathering process used for identifying social presence factors was through retrospectively analysing the archived conference messages to categorise and measure frequency of communication and learning processes. This process involved calculating the frequency of messaging, and analysis of conference message content.

In the first study, the main purpose of the messages had been categorised into a simple three way framework which was extended to include the social presence factors. The analysis framework used a categorisation similar to the first study, but which labelled units of meaning within each message for its primary purpose and content into:

- Cognitive/content, discussion and commentary on the course content

- System messages (relating to learning the FirstClass software, or access issues) and administrative messages and

- Social content analysed into social presence factors using the multiscale. Social Presence categories developed by Rourke et al. (1999) for defining and measuring social presence in a computer conference These are:

- Interactive responses, threaded responses of socially appreciative nature,

- Affective responses expressing emotion, feeling and mood which are expressed by emoticons, humour and self disclosure and

- Cohesive responses which are group activities which build a cohesive group environment. These are measured by factors such as salutations, 
addressing participants by name and addressing the group as 'we', 'our' or 'us'.

\section{FINDINGS OF THE STUDY}

\subsection{The teacher's role in facilitating social presence}

In the early stages, the teacher had an important role helping students establish social presence. In the first weeks, students were required to introduce themselves to the group with specific points of discussion such as professional role, purpose for course choice and previous conferencing experience. Though most students were comfortable with the internet for email and as an information source $(76.5 \%)$, computer conferencing was new to many. Students talked about their feelings of inadequacy entering a space with no visual cues about the other students.

The course was structured so that the teacher modeled social presence factors in the first week of semester. Each introductory message sent by students was responded to encouragingly by the teacher who used that period to teach the students social practice and use of the software elements. Such factors as use of reply and quoting in messages rose in frequency in response to both the teacher's model and the specific directions given about these aspects of the software. Similarly the 'affective' behaviour category of humour, emotion and self disclosure were a style of communication used by the teacher from an early stage and quickly emulated by many students.

By the second week the group began to welcome one another and to practise the social presence factors modelled by the teacher (shown below).

Table 1. Social Presence Factors

\begin{tabular}{|l|r|r|r|r|}
\hline Social Presence & $\begin{array}{l}\text { Affective } \\
\text { units }\end{array}$ & Interactive units & $\begin{array}{l}\text { Cohesive } \\
\text { units }\end{array}$ & Total \\
\hline Week 1Students & 31 & 29 & 33 & 93 \\
\hline Teacher & 4 & 13 & 18 & 35 \\
\hline Total & 35 & 42 & 51 & 128 \\
\hline Week 2 Students & 55 & 117 & 57 & 229 \\
\hline Teacher & 2 & 14 & 3 & 19 \\
\hline Total & 57 & 131 & 60 & 248 \\
\hline Week 3 Students & 15 & 20 & 16 & 51 \\
\hline Teacher & 1 & 6 & 4 & 10 \\
\hline Total & 16 & 26 & 20 & 113 \\
\hline
\end{tabular}




\subsection{Social presence in small group structure}

The structure of the course required task based small group discussions be established in the early stages of the semester. After the introduction phase, students communicated in conference spaces with fewer participants who shared a content focus they had both suggested or chosen. Moving into a small group collaborative environment meant that students could establish small group relationships in a more informal space and this was conducive to social presence comments included in most content messages whatever their complexity. The course required the students to break into subgroups by choice of issue for discussion and then if common issues showed a grouping pattern, the issues discussion groups were used as the basis of forming small collaborative groups for the second collaborative assessment task.

Small group analysis

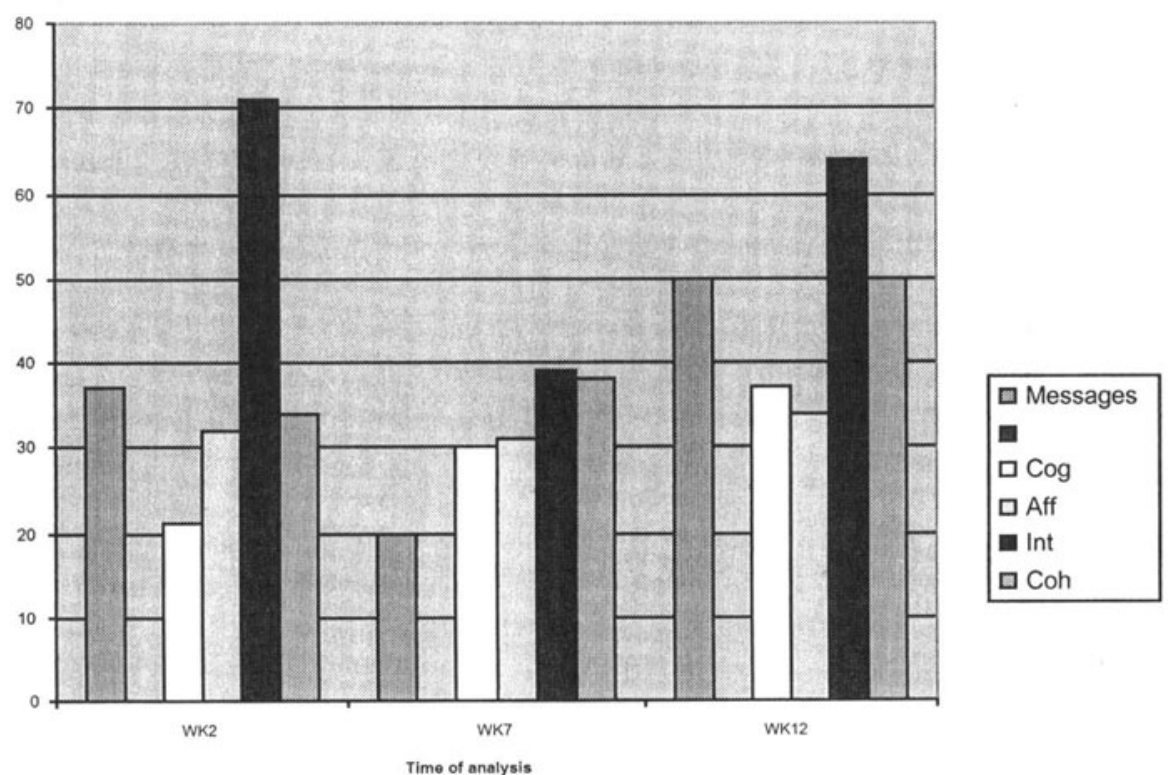

Figure 1. Total of small group factors compared over 3 analysis periods

Their comments continued to project a social presence and group cohesiveness as the groups interacted and the predictions of Walther (1996) 
that groups would work even harder to establish personal relationships online over a long period of time were shown to occur in the analysis of one representative small group shown in Figure 1. Cognitive content also rose over several weeks, reflecting the cognitive focus and purpose of the discussions, but this was accompanied by a continued high level of social presence factors.

These results do not agree with those of Rourke et al. (1999) and Hara, Bonk and Angeli (2000), who found that purely social interaction, not focused on the content of the course, declined as the conference progressed, which they concluded was because students got to know one another better. The conclusions from the analysis of this research study support the argument that social relationships require continuation of social presence factors through a much longer period, as one semester is only the beginning of group formation online.

\subsection{Monitoring small groups and providing multiple layers of communication}

Again, the teacher played an active role in monitoring progress and social cohesion of small groups through the small group discussions. There was some need for multiple layers of communication for effective social presence to be established in some groups and the teacher can organise and suggest communication through phone calls, meetings or synchronous chat as well as through the computer conference. This helped the group to establish the trust and ease of communication for cognitive development to occur. One of the small collaborative groups laboured under misinterpreted social presence until they talked their issues over by phone with the teacher's guidance.

\subsection{Collaborative learning}

As with the first study, collaborative learning was reported as an enhancement of learning. The students described working harder than in other distance subjects because of their accountability to the group and because they engaged more with reading and reflection on a wider range of resources than they would have consulted alone, particularly as they had to contribute to an ongoing discussion. Many described the motivation for learning that group communication provided as the group developed and they described their enjoyment of the interaction which reduced their usual isolation in distance learning or in fact any adult study. The different perspectives provided by the different students was particularly seen as an 
advantage to their learning as it took them out of their own limited view of the subject.

\section{CONCLUSIONS}

Walther's (1996) proposition that relationships require longer to develop in an electronic medium was supported by the study into social presence where even at the end of the semester, members of the groups were motivated to continue their social interaction and their social relationships. Establishing social presence is an important aspect for effective online interaction and learning where the high rate of cognitive analysis of units within messages showed the active engagement students maintained with the cognitive learning of the course. This was accompanied by a continuing high rate of social presence factors establishing the interrelationship of cognitive and social presence online. The teacher's role developing a secure learning environment and modelling social presence factors and continuing to monitor and facilitate conference interaction was a major factor in the success of this interactive process in online learning.

\section{REFERENCES}

Culnan, M. J. and Markus, M. L. (1987) Information technologies. In Handbook of organisational communication: An interdisciplinary perspective, F. M. Jablin, L. Putnam, K. H. Roberts and L. W. Porter (eds.), Newbury Park, CA. (Sage cited in Walther (1996)).

Forman, E. A. and McPhail, J. (1993) Vygotskian perspective on children's collaborative problem-solving activities. In Contexts for Learning: Sociocultural Dynamics in Children's Development, E. A. Forman, N. Minick and C. A. Stone (eds.), Oxford University Press, New York, pp. 213-229.

Garrison, D. R. (1997a) Computer conferencing and distance education: cognitive and social presence issues. In The new learning environment: A global perspective, Proceedings of the ICDE World Conference, Pennsylvania State University.

Garrison, D. R (1997b) Computer conferencing: the post-industrial age of distance education. Open Learning, June, pp. 3-11.

Gunawardena, C. N. and Zittle, F. (1997) Social presence as a predictor of satisfaction within a computer-mediated conferencing environment. American Journal of Distance Education, 11,3 , pp. 8-26.

Hara, N., Bonk, C. J. and Angeli, C. (2000) Content Analysis of Online Discussion in an Applied Educational Psychology. Instructional Science, 28 (2), pp. 115-152.

Lindlif, T. R. and Shatzer, M. J. (1998) Media ethnography in virtual space: strategies, limits, and possibilities. Journal of Broadcasting and Electronic Media, Spring, 42, 2, pp. 170189.

Parks, M. R. and Floyd, K. (1996) Making friends in cyberspace. Joumal of communication, 46 (1), pp. 80-90. 
Rice, R. E. (1993) Media appropriateness: Using social presence theory to compare traditional and new organisational media. Human Communication Research, 19, pp. 451-484. (Quoted in Lindlif and Shatzer (1998)).

Rourke, L., Anderson T., Garrison, D. R. and Archer, W. (1999) Assessing social presence in asynchronous text-based computer conferencing. Canadian Journal of Distance Education, 14, 2, pp. 50-71.

Stacey, E. (1999) Collaborative learning in an online environment. Canadian Journal of Distance Education, 14, 2, pp. 14-33.

Vygotsky, L. S. (1978) Mind in Society: The Development of Higher Psychological Processes. (Cole, M. M., Lopez-Morillas, Luria, A. R and Wertsch, J. (trans.)), Harvard University Press, Cambridge, MA.

Walther, J. B. (1993) Impression development in computer mediated interaction. Western Journal of Communication, 57, pp. 381-398.

Walther, J. B. (1996) Computer-mediated communication: Impersonal, interpersonal, and hyperpersonal interaction. Communication Research, 23 (1), pp. 3-41.

Walther, J. B. and Burgeon, J. K. (1992) Relational communication in computer mediated interaction. Human Communication Research, 19, pp. 50-88.

\section{BIOGRAPHY}

Dr. Elizabeth Stacey is a Senior Lecturer in the Education Faculty at Deakin University. She teaches pre-service teacher education studies, postgraduate distance education coursework and supervises students researching flexible learning and computer and communication technologies. Her research and publications are focused on these areas including learning with computers, and the use of interactive technologies, particularly audiographics, interactive television and computer conferencing. 